\title{
Design and performance evaluation of a coplanar multimodality scanner for rodent imaging
}

\author{
E Lage ${ }^{1}$, J J Vaquero ${ }^{1}$, A Sisniega ${ }^{1}$, S España ${ }^{2}$, G Tapias ${ }^{1}$, M Abella ${ }^{1}$, \\ A Rodríguez-Ruano ${ }^{1}$, J E Ortuño ${ }^{3,4}$, A Udías ${ }^{5}$ and M Desco ${ }^{1}$ \\ ${ }^{1}$ Unidad de Medicina y Cirugía Experimental, Hospital General Universitario Gregorio \\ Marañón, Madrid, Spain \\ ${ }^{2}$ Departamento de Física Atómica, Molecular y Nuclear, Facultad de Ciencias Físicas, \\ Universidad Complutense, Madrid, Spain \\ ${ }^{3}$ Networking Research Center on Bioengineering, Biomaterials and Nanomedicine \\ (CIBER-BBN), Zaragoza, Spain \\ ${ }^{4}$ Department of Electronic Engineering, ETSIT, Universidad Politécnica de Madrid, Madrid, \\ Spain \\ 5 Departamento de Estadística e Investigación Operativa. Universidad Rey Juan Carlos, \\ Fuenlabrada, Spain \\ E-mail: elage@mce.hggm.es
}

Received 6 April 2009, in final form 14 July 2009

Published 21 August 2009

Online at stacks.iop.org/PMB/54/5427

\begin{abstract}
This work reports on the development and performance evaluation of the $\mathrm{VrPET} / \mathrm{CT}$, a new multimodality scanner with coplanar geometry for in vivo rodent imaging. The scanner design is based on a partial-ring PET system and a small-animal CT assembled on a rotatory gantry without axial displacement between the geometric centers of both fields of view (FOV). We report on the PET system performance based on the NEMA NU-4 protocol; the performance characteristics of the CT component are not included herein. The accuracy of inter-modality alignment and the imaging capability of the whole system are also evaluated on phantom and animal studies. Tangential spatial resolution of PET images ranged between $1.56 \mathrm{~mm}$ at the center of the FOV and 2.46 at a radial offset of $3.5 \mathrm{~cm}$. The radial resolution varies from $1.48 \mathrm{~mm}$ to $1.88 \mathrm{~mm}$, and the axial resolution from $2.34 \mathrm{~mm}$ to $3.38 \mathrm{~mm}$ for the same positions. The energy resolution was $16.5 \%$ on average for the entire system. The absolute coincidence sensitivity is $2.2 \%$ for a $100-700 \mathrm{keV}$ energy window with a $3.8 \mathrm{~ns}$ coincident window. The scatter fraction values for the same settings were $11.45 \%$ for a mouse-sized phantom and $23.26 \%$ for a rat-sized phantom. The peak noise equivalent count rates were also evaluated for those phantoms obtaining $70.8 \mathrm{kcps}$ at $0.66 \mathrm{MBq} / \mathrm{cc}$ and $31.5 \mathrm{kcps}$ at $0.11 \mathrm{MBq} / \mathrm{cc}$, respectively. The accuracy of inter-modality alignment is below half the PET resolution, and the image quality of biological specimens agrees with measured performance parameters. The assessment presented in this study shows that the VrPET/CT system is a good performance small-animal imager, while the
\end{abstract}


cost derived from a partial ring detection system is substantially reduced as compared with a full-ring PET tomograph.

(Some figures in this article are in colour only in the electronic version)

\section{Introduction}

The increasing number of animal models of human diseases and the radiolabeling of biologically significant molecules have made small-animal PET a valuable tool in medical research (Phelps 2000, Pomper 2001, Weissleder 2002). New clinical scanners combine the functional information of PET or SPECT images with an anatomical imaging modality such as $\mathrm{X}$-ray computerized tomography whose fusion improves the image interpretation (Townsend and Beyer 2002). Given the relevance of murine models in biomedical research, this same approach is being adopted in small-animal dedicated tomographs, and several multimodality systems (including MRI/PET) are being investigated, developed and introduced to the market (Meei-Ling et al 2006, Liang et al 2007, Raylman et al 2007, Cherry et al 2008). Although there have been recent promising approaches for the design of combined PET/CT detectors (Berard et al 2005, Fontaine et al 2005, Riendeau et al 2008, Nassalski et al 2008), most common implementations align two essentially independent imaging systems in the axial direction with little or no mechanical integration. A motorized patient bed enables multimodality imaging to be performed with an axial translation. Subsequently, the registration and fusion of the two image sets are done based on precalculated alignment parameters. The PET/CT scanner presented in this work (VrPET/CT) uses an alternative geometry consisting of a partial ring of PET detectors and a small-animal CT system assembled on a rotatory gantry in such a way that there is no axial displacement between the geometric centers of both fields of view (figure 1(a)). This system was developed by the Unidad de Medicina Experimental of the Gregrorio Marañon Hospital (Madrid, Spain) and is now manufactured by SEDECAL (Madrid, Spain). The PET component of this scanner is based on a partial ring PET tomograph called rPET, previously developed at our institution (Vaquero et al 2005). This system consisted of four detector heads arranged as two orthogonal pairs of diagonally opposed detectors, providing a field of view (FOV) of approximately $45 \times 45 \times 45 \mathrm{~mm}^{3}$ and an absolute sensitivity of $1 \%$ per detector pair (Cañadas et al 2008). The development of the VrPET/CT was motivated by the idea of integrating the rPET system with a micro-CT, simultaneously improving some functional and technological features. In this new configuration the PET scanner geometry was modified by reallocating the four detectors into two wider V-shaped blocks (figure 1). This geometry enables each detector to accept coincident photons with its two opposed detectors rather than with only one as it was in the rPET, thus obtaining an extended transaxial FOV.

This document focuses on the design and integration of the VrPET system as well as on the performance evaluation of this coplanar scanner for small-animal imaging purposes. The work includes measurements of spatial resolution, sensitivity, scatter fraction, counting rate and image quality based on the recently approved NEMA NU-4 standard (NEMA 2008). The accuracy of inter-modality alignment and the imaging capability of the whole system are also evaluated by phantom and animal studies. The implementation details and performance evaluation of the micro-CT system have already been detailed elsewhere (Lage et al 2006, Vaquero et al 2008). 

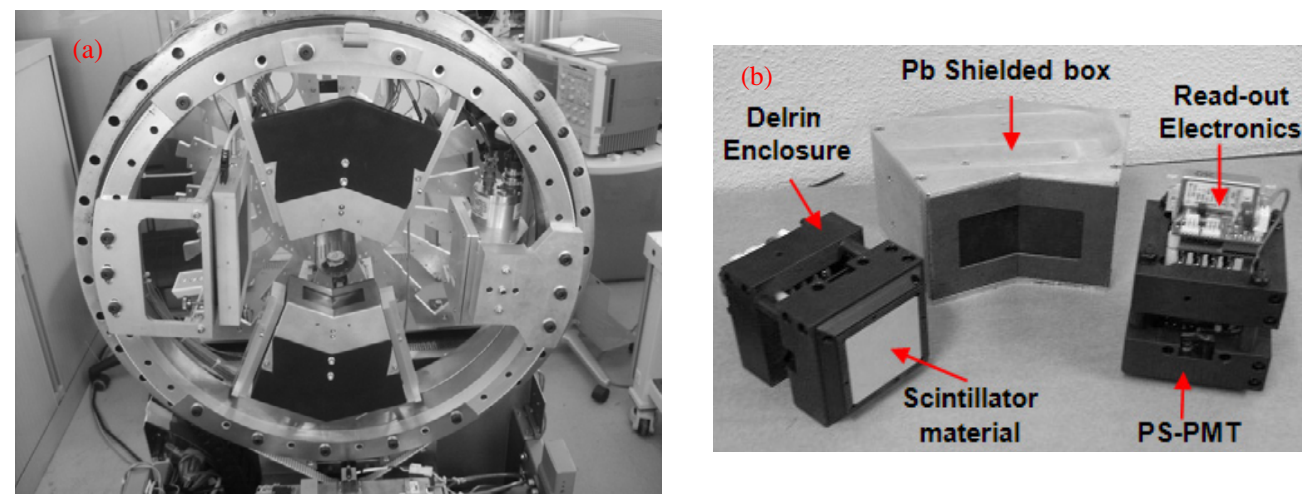

Figure 1. (a) VrPET/CT gantry implementation: the system consists of a rotatory gantry containing four PET detectors arranged into two V-shaped blocks and a small-animal CT scanner composed by a flat panel detector (right) and a micro-focus x-ray tube (left); the centers of the field of view for both modalities are intrinsically aligned. (b) VrPET detector modules: each module contains two single detectors packed in black delrin enclosures which fit in a light tight lead $(\mathrm{Pb})$ shielded box. Read-out electronics together with an additional timing stage for the last dynode signal and a high voltage supply are integrated in a compact PCB stack directly attached to the PS-PMT sockets.

\section{Materials and methods}

\subsection{System description}

The system uses two double-detector modules, each of them comprised two single detectors based on a $30 \times 30$ lutetium-yttrium oxyorthosilicate (LYSO) crystal array coupled to a position-sensitive photomultiplier (PS-PMT, Hamamatsu H8500). The scintillator matrices are assembled using individual crystals with dimensions $1.4 \times 1.4 \times 12 \mathrm{~mm}^{3}$ separated by a $100 \mu \mathrm{m}$ thick white reflector layer to improve the light collection (Vaquero et al 1998). The photomultipliers have 12 stages of metal channel dynode and $8 \times 8$ multiple anodes, providing an active area of $49 \mathrm{~mm}^{2}$. The material used for coupling the PMT and the crystal array was optical grease (BC-630 Bicron/Saint Gobain). Read-out electronics, together with an additional timing stage for the last dynode signal and a high voltage supply, are integrated in a compact PCB stack directly attached to the PS-PMT sockets (figure 1(b)). Last dynode signals of these detectors are fed into pulse discriminator circuits to generate digital timing signals to detect coincident scintillations occurring within a coincidence window of $3.8 \mathrm{~ns}$. The detection of a valid photon pair triggers the digitization of the position and energy signals necessary to characterize the event.

Resulting data from analog-to-digital converters are sent to the acquisition computer via a PCI communication interface. Additionally, the acquisition system includes a counter module with eight general-purpose scalers. The current values of these registers are included in the data frame for each detected coincidence, thus providing additional synchronization information such as the detectors position, overall elapsed time and gating signals. The complete processing of each event (including digitization and transfer from ADCs to the communication module) takes roughly $1.8 \mu \mathrm{s}$, leading to a maximum processing throughput of about $500 \mathrm{Kcounts} \mathrm{s}^{-1}$.

The detectors are mounted using black delrin enclosures which fit in a V-shaped shielded box that attaches the detectors to the gantry (figure 1(b)). These modules are positioned at a $140 \mathrm{~mm}$ center-to-center distance to form 30 partial rings with a $1.5 \mathrm{~mm}$ pitch. The angle in 
Table 1. Summary of VrPET scanner characteristics.

\begin{tabular}{ll}
\hline Detectors & LYSO \\
Crystal material & $1.4 \mathrm{~mm} \times 1.4 \mathrm{~mm} \times 12 \mathrm{~mm}$ \\
Crystal size & $1.5 \mathrm{~mm}$ \\
Crystal pitch & Flat panel PS-PMT (Hamamatsu H8500) \\
Photomultiplier type & $3600(900$ per single detector) \\
Number of crystals & 4 \\
Number of PMTs & \\
Gantry and geometry & $86.6 \mathrm{~mm}$ \\
Transaxial FOV & $45.6 \mathrm{~mm}$ \\
Axial FOV & $180^{\circ}$ \\
Detectors rotation & $4.5-18^{\circ} \mathrm{s}^{-1}$ \\
Rotation speed (standard- maximum) & Persistent scope mode or CT scout \\
Sample positioning & $275 \mathrm{~mm}$ horizontal $/ 35 \mathrm{~mm}$ vertical \\
Bed travel & \\
\hline
\end{tabular}

the transaxial plane between the detectors of the same block $\left(143.5^{\circ}\right)$ was selected to provide a transaxial FOV that matches the CT one, while allowing the $\mathrm{x}$-ray cone beam to illuminate the x-ray detector surface without shadows. The resulting FOV of the PET subsystem is $86.6 \mathrm{~mm}$ diameter in the transaxial direction and $46.5 \mathrm{~mm}$ in the axial. The scanner also includes a linear motion stage attached to the bed to move the sample along the axial FOV for whole body studies. Both motion axes (rotating gantry and bed) are controlled by stepper motors and digital drives connected to the central processing unit which also controls the different system components during PET or CT data acquisitions.

PET scans are done by continuously rotating the detectors $180^{\circ}$ clockwise and counterclockwise alternatively, in order to acquire complete datasets for the scanned volume. The rotation velocity is set by default to $4.5^{\circ} \mathrm{s}^{-1}$; this speed is a design parameter derived from the expected absolute sensitivity that results in minimum frame duration of $40 \mathrm{~s}$. However, the mechanical and control components of the tomograph are dimensioned to be able to rotate the detectors up to $18^{\circ} \mathrm{s}^{-1}$, thus enabling a minimum frame duration of $10 \mathrm{~s}$ if desired. In addition, the scanner is able to acquire $2 \mathrm{D}$ projection views at fixed detector locations. In this mode high temporal and high spatial resolution 2D images can be acquired (Siegel et al 1999). This feature has been used to implement a persistence scope mode, useful for interactive centering of the sample within the FOV and to obtain complementary data for dynamic imaging studies, by providing a high temporal resolution projection image sequence of the initial passage of the tracer (Zingone et al 2002).

Data acquisition, processing, reconstruction, fusion and image viewing are managed from a remote computer which runs an IDL-based console that includes user interface and analysis tools (Pascau et al 2006). A summary of the VrPET characteristics is reported in table 1.

\subsection{Data processing and correction procedures}

During PET scans the acquisition computer processes the digitized signals from the detectors to calculate the interaction point and the energy of the two detected gamma photons. This information is recorded in one or more list mode files on an event-by-event basis. Before reconstruction, the position and energy values are mapped to individual crystals by means of a look-up table (LUT) previously computed, and the energy value for each event is used to construct the corresponding crystal spectrum. These data are binned into sinograms consisting of 117 radial bins and 190 angular bins $\left(180^{\circ}\right.$ rotation) for each of the direct and oblique crystal 
combinations. During this process other corrections are also applied to the data: energy discrimination and corrections for decay, normalization, detectors alignment (Abella et al 2006) and dead time. The normalization correction is based on a previous measurement of a ${ }^{68} \mathrm{Ge}$ uniformity phantom. A normalization 3D sinogram derived from this measurement is used to correct the data sinograms before applying any analytic reconstruction protocol. For all the experiments described in this work, these normalization factors were calculated from a $12 \mathrm{~h}$ acquisition containing more than 100 Mcounts.

For $2 \mathrm{D}$ reconstructions, the sinograms are converted to 59 slices by Fourier rebinning (FORE) or single slice-rebinning (SSRB) algorithms with a configurable span and maximum ring difference. The $2 \mathrm{D}$ sinograms can be reconstructed with standard filtered backprojection (FBP) or with a fast iterative 2D OSEM algorithm (Ortuño et al 2006, 2008). A 3D OSEM reconstruction algorithm has also been adapted to this PET imager (Herráiz et al 2006).

\subsection{NEMA NU-4 performance measurements}

This performance evaluation has been structured according to the recently approved NEMA NU-4 standard (NEMA 2008) for the assessment of small-animal positron emission tomographs. PET system parameters evaluated were spatial and energy resolution, sensitivity, count rate, scatter fraction and image quality. All these measurements were carried out with a $3.8 \mathrm{~ns}$ coincidence window and a $100-700 \mathrm{keV}$ energy window.

2.3.1. Spatial resolution. The spatial resolution of the system was assessed with an encapsulated ${ }^{22} \mathrm{Na}$ point source with a diameter of $0.3 \mathrm{~mm}$ and activity of $1.1 \mathrm{MBq}$. Different measurements were taken in the central slice of the axial FOV at several radial distances from the geometrical center (from $z=0.0 \mathrm{~mm}$ to $z=35 \mathrm{~mm}$ using $5 \mathrm{~mm}$ increments). Resolution at the same transaxial points was also evaluated at $\frac{1}{4}$ axial FOV $(z=11.4 \mathrm{~mm})$. The scan period was $2.0 \mathrm{~min}$ at each position and 2-3 million counts were typically collected per scan. The list mode was rebinned into 2D sinograms by SSRB and reconstructed by FBP with a ramp filter (voxel size: $0.74 \times 0.74 \times 0.77 \mathrm{~mm}^{3}$ ). The spatial resolution was calculated as the full width at half maximum (FWHM) of the profiles in the radial, axial and tangential directions. The measurement of all mentioned data points was replicated at least once.

2.3.2. Sensitivity. System sensitivity was evaluated using the same source as in the previous test, carefully centered in axial and transaxial directions. Two-minute acquisitions were obtained while the source was being stepped in $0.77 \mathrm{~mm}$ increments across the scanner from end to end of the axial FOV. The activity was low enough so that dead-time losses were negligible. The sinogram for each axial position was rebinned using SSRB in order to assign the counts present in the oblique lines of response (LORs) to the image slice where the LOR crosses the scanner axis. For each row of each sinogram, the highest value was located and all pixels further than $1 \mathrm{~cm}$ from this peak were set to zero. The resulting counts in each sinogram were added to obtain the total counts per slice. Slice sensitivity was calculated by dividing the total counts per slice by the activity present in the source and the resulting values were corrected to consider the branching ratio of the ${ }^{22} \mathrm{Na}$. This procedure was replicated using three different energy windows: 100-700, 250-650 and 400-700 keV.

2.3.3. Energy resolution. During the calibration procedure of the scanner, the energy spectra for the 3600 crystals were acquired within an energy window of 100-700 keV. The resulting spectra were analyzed for the $511 \mathrm{keV}$ peak and a Gaussian function was fitted to each peak. 
Energy resolutions were calculated as the FWHM (in keV) of the Gaussian curves divided by the photopeak energy, in percentage. A whole system energy resolution was also calculated using an 'average spectrum' formed by aligning and scaling the individual spectra of all crystals to a common reference.

2.3.4. Count rate performance. Mouse- and rat-sized NEMA test phantoms were utilized in these measurements. Both phantoms were high-density polyethylene cylinders with dimensions in proportion to the respective animal sizes $(25 \mathrm{~mm}$ diameter, $70 \mathrm{~mm}$ long for the mouse-sized phantom and $50 \mathrm{~mm}$ diameter, $150 \mathrm{~mm}$ long for the rat-sized phantom). Cylindrical holes (3.2 mm diameter) were drilled parallel to the central axis in both phantoms at radial distances of $10 \mathrm{~mm}$ (mouse) and $17.5 \mathrm{~mm}$ (rat). The test phantom line source insert was a flexible tube with an outside diameter which fits on the $3.2 \mathrm{~mm}$ hole and with a fillable section $10 \mathrm{~mm}$ shorter that the axial extent of the phantoms. Prior to each acquisition, the phantom was positioned on the animal bed and centered in the transverse and axial fields of view. The initial activity concentration was $1.1 \mathrm{MBq} \mathrm{cc}^{-1}$ (37.92 $\mathrm{MBq}$ total activity) for the mouse-sized and $0.19 \mathrm{MBq} \mathrm{cc}^{-1}(55.87 \mathrm{MBq}$ total activity) for the rat-sized phantom ( $\left.{ }^{18} \mathrm{~F}-\mathrm{FDG}\right)$. The data were acquired for $12 \mathrm{~h}$ in $20 \mathrm{~min}$ frames in both cases, and sinograms for each acquisition frame and slice were generated by SSRB. These data were analyzed following the guidelines described in the NEMA NU-4 protocol to estimate prompt (total counts), true, scatter and random count rates for each frame, as a function of the average activity concentration $\left(\mathrm{kBq} \mathrm{cc}^{-1}\right)$ in the entire phantom volume (34.4 $\mathrm{cc}$ for the mouse-sized and $294.5 \mathrm{cc}$ for the rat-sized phantom). The noise equivalent count rates (NEC rates) were obtained using the following expression:

$$
\mathrm{NEC}_{\text {rate }}=\frac{\text { True }_{\text {rate }}^{2}}{\text { Total }_{\text {rate }}}
$$

2.3.5. Scatter fraction. Scatter fraction (SF) was analyzed for the rat- and mouse-sized phantoms using the last five frames of the previous acquisitions. Due to the low activity concentration inside the FOV, these frames contained random event and count loss rates below $1.0 \%$ of the true event rate. In these conditions it is assumed that the number of random counts is negligible and the difference between true and prompt count rates is only due to scattered events. Relative system sensitivity to scattered radiation (scatter fraction, SF) was obtained by aligning the projection angles of each sinogram in such a way that the maximum pixel value in each projection (center of the line source) became aligned with the central pixel of the sinogram. After this process and for every frame, a sum projection in the angular direction was obtained for each slice.

SF was then calculated by taking the number of scattered events in the summed projection and dividing this number by the total number of events in the profile. The number of scattered events was determined using a $14 \mathrm{~mm}$ window around the maximum radial bin. This number was obtained as the sum of the events outside the window and the events within this window below a straight line through the bins at $\pm 7 \mathrm{~mm}$ of the maximum radial bin. The system scatter fraction was computed as the weighted average of the scatter fraction obtained for each slice and frame.

2.3.6. Uniformity, recovery coefficients and spillover ratios. The quantitative accuracy of the system was evaluated by imaging the image quality phantom (IQ) defined by NEMA NU-4 (figure 2(a)). We filled the main compartment of this phantom with a water solution of ${ }^{18} \mathrm{~F}$ containing a total activity of $3.06 \mathrm{MBq}$. With respect to the two cold compartments attached to 

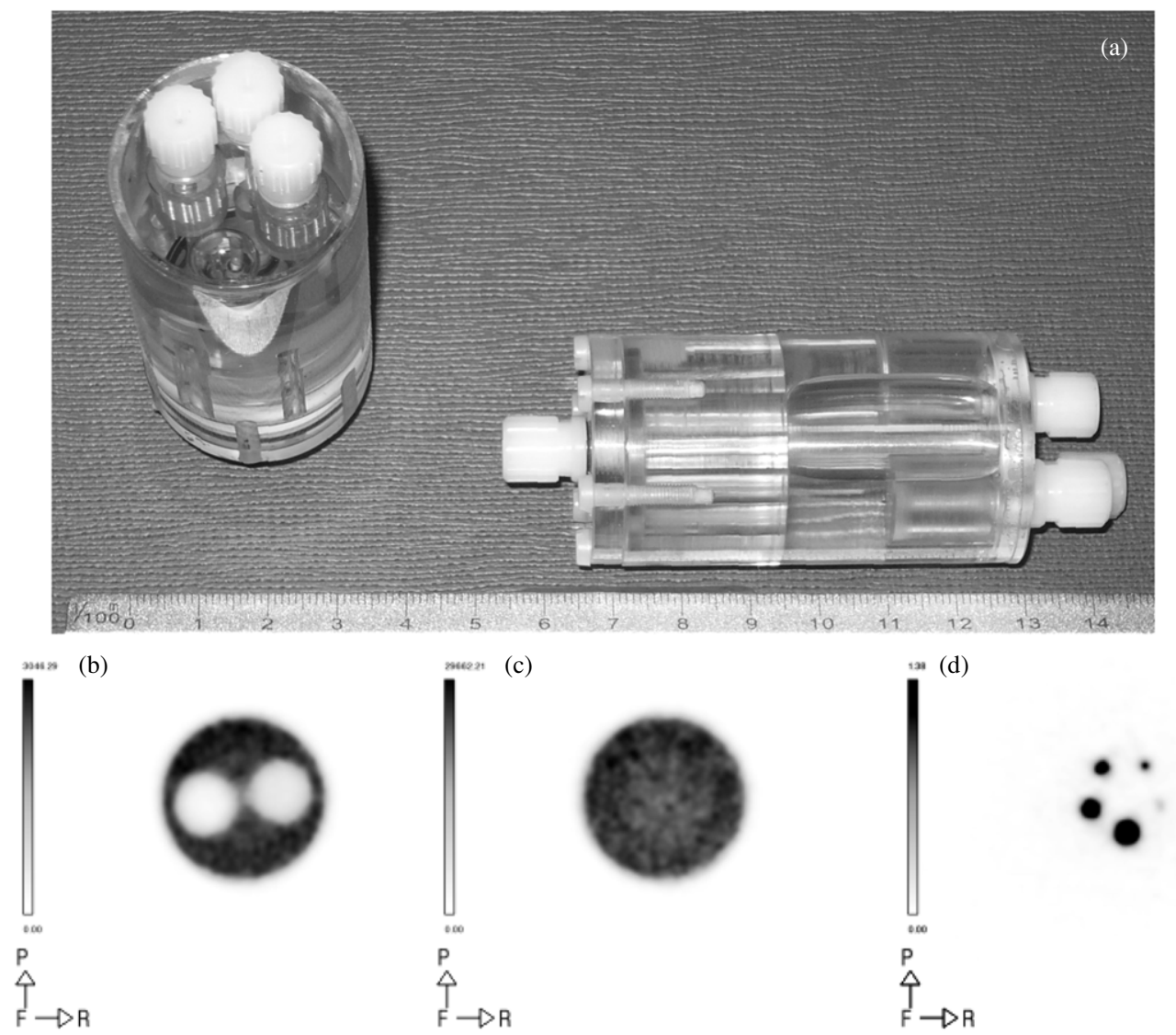

Figure 2. IQ phantom photograph (a) and FBP reconstructed slices (bottom) showing the cold inserts (b), uniform (c) and hot rods region (d). Rods in the right slice are of 5, 4, 3, 2 and $1 \mathrm{~mm}$ in diameter.

the lid of the phantom (figure 2(b)), one was filled with water and the other was left empty. The phantom was scanned for $20 \mathrm{~min}$ and sinograms were generated with the acquired data. The image uniformity, recovery coefficient values and quantitative accuracy of data were measured on the resulting images. The noise in the uniform region of the phantom is indicative of the signal to noise ratio performance of the imaging system, while the uniformity in this region is a measure of the attenuation and scatter effects. Uniformity was evaluated by drawing a $22.5 \mathrm{~mm}$ diameter by $10 \mathrm{~mm}$ long cylindrical volume of interest (VOI) over the center of the uniform region of the IQ phantom (figure 2(c)). The true isotope concentration was taken to be the mean value of this uniform region. Recovery coefficients (RC) were measured on the image slices covering the central $10 \mathrm{~mm}$ length of the rods. Slices were averaged to obtain a single slice of lower noise (figure 2(d)), and circular regions of interest (ROIs) were drawn around each rod with diameters twice the physical diameter of the rod. The transverse image pixel coordinates of the location with the maximum ROI values were recorded and used to create line profiles along the rods in the axial direction. The mean pixel value measured along each of those profiles was taken as the best estimation of isotope concentration in the rods. The standard deviation of the resulting recovery coefficient was calculated as follows: 


$$
\% \mathrm{STD}_{\mathrm{RC}}=100 * \sqrt{\left(\frac{\mathrm{STD}_{\text {lineprofile }}}{\text { Mean }_{\text {lineprofile }}}\right)^{2}+\left(\frac{\mathrm{STD}_{\text {background }}}{\text { Mean }_{\text {background }}}\right)^{2}} .
$$

Scatter effects in reconstructed images were measured by defining VOIs in the water- and air-filled cylindrical inserts of the IQ phantom (figure 2(b)). The diameter of each VOI was $4 \mathrm{~mm}$ and encompassed the central $7.5 \mathrm{~mm}$ in length. The ratio of the mean in each cold region to the mean of the hot uniform area was reported as spillover ratio (SOR).

For the analysis, images of the IQ phantom were reconstructed using all available reconstruction algorithms with the following settings: FBP reconstruction (pixel size $0.74 \mathrm{~mm}$, slice thickness $0.77 \mathrm{~mm}$ ) was smoothed with a Butterworth filter (cut-off frequency 0.43 , order 12); the 2D OSEM images (pixel size $0.43 \mathrm{~mm}$, slice thickness 0.77 ) were reconstructed using 10 subsets with 5 iterations and the 3D OSEM images (pixel size $0.43 \mathrm{~mm}$, slice thickness $0.77 \mathrm{~mm}$ ) were reconstructed using 40 subsets with a single iteration. Prior to apply 2D reconstruction algorithms sinograms were rebinned using SSRB with an axial difference of 15 crystals.

\subsection{Phantom and animal studies}

Although it is not included in the NEMA protocol, we also evaluated the imaging capabilities of the system by scanning a hot rods Derenzo phantom. The data were acquired for $180 \mathrm{~min}$ in a single bed position; the total activity in the phantom at the beginning of the acquisition was $5.2 \mathrm{MBq}\left({ }^{18} \mathrm{~F}-\mathrm{FDG}\right)$. This phantom has six groups of rods with diameter of $4.8 \mathrm{~mm}$, $4.0 \mathrm{~mm}, 3.2 \mathrm{~mm}, 2.4 \mathrm{~mm}, 1.6 \mathrm{~mm}$ and $1.2 \mathrm{~mm}$; the separation between rod centers is twice the rod diameter.

Finally, in vivo studies on a $245 \mathrm{~g}$ Wistar rat with induced ischemia in the left side of the brain and on a $32 \mathrm{~g} \mathrm{BALB} / \mathrm{c}$ mouse were carried out in order to demonstrate the imaging capabilities of the VrPET/CT scanner. The rat was intravenously injected with $59.9 \mathrm{MBq}$ of FDG, and the mouse with 16.2 MBq of the same radiopharmaceutical. In both cases PET scanning started $60 \mathrm{~min}$ after the injection and data were acquired for $25 \mathrm{~min}$ per bed position (three positions for both animals). After each PET acquisition, a CT image was acquired using a low-dose and high-speed acquisition protocol: a single bed focused on the head for the rat and two bed positions for the mouse (whole body study). The pixel size was $200 \mu \mathrm{m}$ in both cases and $\mathrm{x}$-ray source settings were $40 \mathrm{kV}, 130 \mu \mathrm{A}$ for the mouse and $45 \mathrm{kV}, 250 \mu \mathrm{A}$ for the rat. PET studies were reconstructed with 3D OSEM, using 40 subsets with a single iteration. CT studies were reconstructed using a FDK algorithm adapted to the specific geometry of the CT scanner (Vaquero et al 2008).

\subsection{Inter-modality alignment}

The physical alignment of both FOVs can be mechanically adjusted only to a certain degree. To achieve higher precision, a geometric transformation matrix between the PET and CT coordinate systems is calculated in a last calibration step, using a simple phantom based on three glass capillaries filled with ${ }^{18}$ F-FDG. These capillaries are arranged in a non-coplanar triangular geometry, each one at a different height ( $y$ coordinate). The static offsets and rotations between both image volumes are computed with an algorithm based on automatic line detection and localization of the corresponding points between the lines on both modalities (Rodriguez-Ruano et al 2008). The resulting values were used on the subsequently acquired scans to enable automatic registration and joint visualization of the images.

Additionally, and with the purpose of evaluating the alignment precision against the layout of the capillaries, this acquisition was repeated five times varying the geometric 

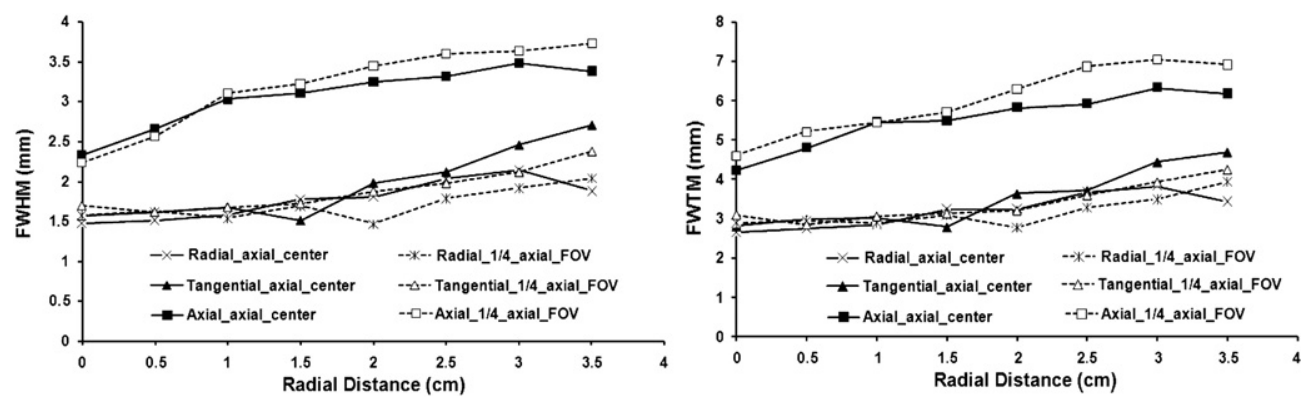

Figure 3. Spatial resolution measured from reconstructed images. Radial, tangential and axial spatial resolution values (FWHM and FWTM) as a function of radial offset. Measurements were done at the axial center of the FOV and at $11.4 \mathrm{~mm}$ from the axial FOV center ( $\frac{1}{4}$ axial FOV).

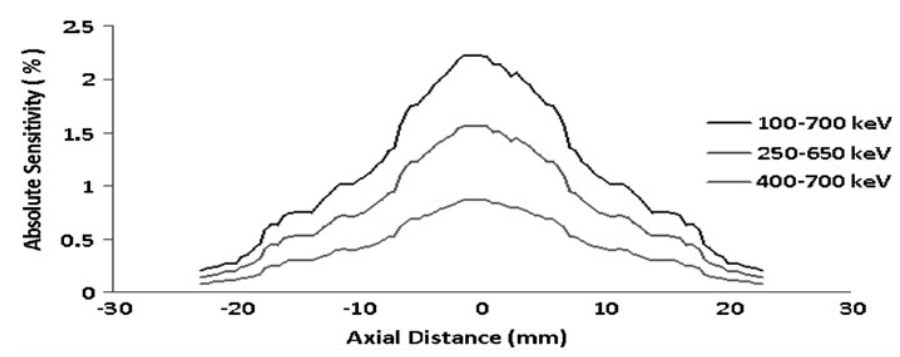

Figure 4. Sensitivity of the VrPET scanner. The slice absolute sensitivity is plotted as a function of the coordinate along the axial axis of the scanner for three typical energy windows.

relations (angles and distances) between capillary sources. To determine registration accuracy, two additional ${ }^{22} \mathrm{Na}$ point sources were acquired simultaneously with each study. After the alignment calculations, the transformation obtained was applied to the data and the positioning accuracy was measured in the point sources (note that these sources were ignored during the registration process).

\section{Results}

\subsection{NEMA NU-4 performance measurements}

3.1.1. Spatial resolution. The axial and transaxial resolutions (FWHM and FWTM) are plotted in figure 3 as a function of the radial distance from the center. The tangential spatial resolution of the system ranged between $1.56 \mathrm{~mm}$ at the center of the FOV and 2.46 at a radial offset of $3.5 \mathrm{~cm}$. The radial resolution ranged from 1.48 to 1.88 , and the axial FWHM increased from $2.34 \mathrm{~mm}$ in the center to $3.38 \mathrm{~mm}$ for the same radial offset. Resolution at the same transaxial points was also evaluated at $\frac{1}{4}$ axial FOV without noticeable variations (figure 3 ). The volumetric resolution computed as the product of the axial, radial and transaxial resolution is $5.4 \mu \mathrm{l}$ in the central FOV increasing to $16.3 \mu \mathrm{l}$ at a $35 \mathrm{~mm}$ radial offset.

3.1.2. Sensitivity. Absolute sensitivity values along the axial FOV are presented in figure 4. The peak sensitivity at the central FOV is $2.22 \%$ for a $100-700 \mathrm{keV}$ energy window, $1.56 \%$ for a $250-650 \mathrm{keV}$ energy window and $0.94 \%$ for the $400-700 \mathrm{keV}$ energy range. 

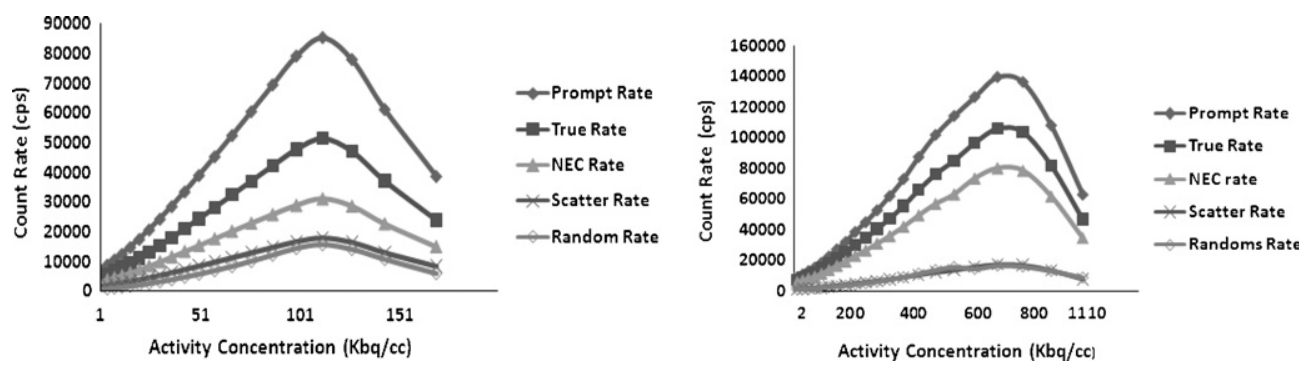

Figure 5. Count rate capability of the VrPET system as a function of the line source activity divided by the total volume of the phantom. Energy window was set at 100-700 keV in both cases (left, rat-sized phantom; right, mouse-sized phantom).

Table 2. Uniformity analysis (arbitrary units).

\begin{tabular}{lcccc}
\hline & Mean & Max. & Min. & $\%$ Std \\
\hline FBP & 3.54 & 5.54 & 1.45 & 16.85 \\
2D OSEM & 3.51 & 5.28 & 1.52 & 16.57 \\
3D OSEM & 2.86 & 4.99 & 1.33 & 15.35 \\
\hline
\end{tabular}

3.1.3. Energy resolution. System energy resolution calculated using an 'average spectrum' of the aligned and scaled individual spectra was $16.5 \%$ with a standard deviation of 2.2 . The worst crystal energy resolution was $33.4 \%$ (outlier crystals on the edge), while the best one was $9.7 \%$.

3.1.4. Count rate performance. Figure 5 shows the behavior of the prompt, true, random, scatter and NEC count rates as a function of the activity concentration in the mouse- and rat-sized phantoms. Prompt counts are the total counts acquired for the system, and the true rate refers to the total coincident event rate minus the scattered and random event rates. The coincidence background noise due to the intrinsic radioactivity contained in LYSO crystals was not subtracted from the measured data since it is negligible in this scanner (less than $4 \mathrm{cps}$ ) due to the low volume of LYSO in the detectors, the narrowness of the coincidence window and scanner calibration settings. The resulting peak NEC rates are $31.5 \mathrm{Kcps}$ at $0.116 \mathrm{MBq}$ $\mathrm{cc}^{-1}$ activity concentration (10.55 MBq within the entire FOV) for the rat-sized phantom and $70.8 \mathrm{Kcps}$ at $0.66 \mathrm{MBq} \mathrm{cc}^{-1}$ activity concentration (17.25 MBq within the entire FOV) for the mouse-sized phantom.

3.1.5. Scatter fraction. As expected, the scatter contribution was found to be significantly lower for the measurement on the mouse phantom than for the rat phantom. The measured SF is $23.26 \%$ for rat-sized objects and $11.45 \%$ for mouse-sized objects.

3.1.6. Uniformity, recovery coefficients and spillover ratios. The uniformity test results are summarized in table 2 . The average pixel value (proportional to activity concentration), the maximum and minimum values and the percentage standard deviation in the VOI defined in the uniform region of the IQ phantom, were measured and reported for each reconstruction algorithm. The recovery coefficients are plotted in figure 6 as a function of the rod diameter. Due to the physical limitations in producing hot spheres in non-zero background with physical walls much thinner than the spatial resolution of the imaging systems, fillable rods of different 

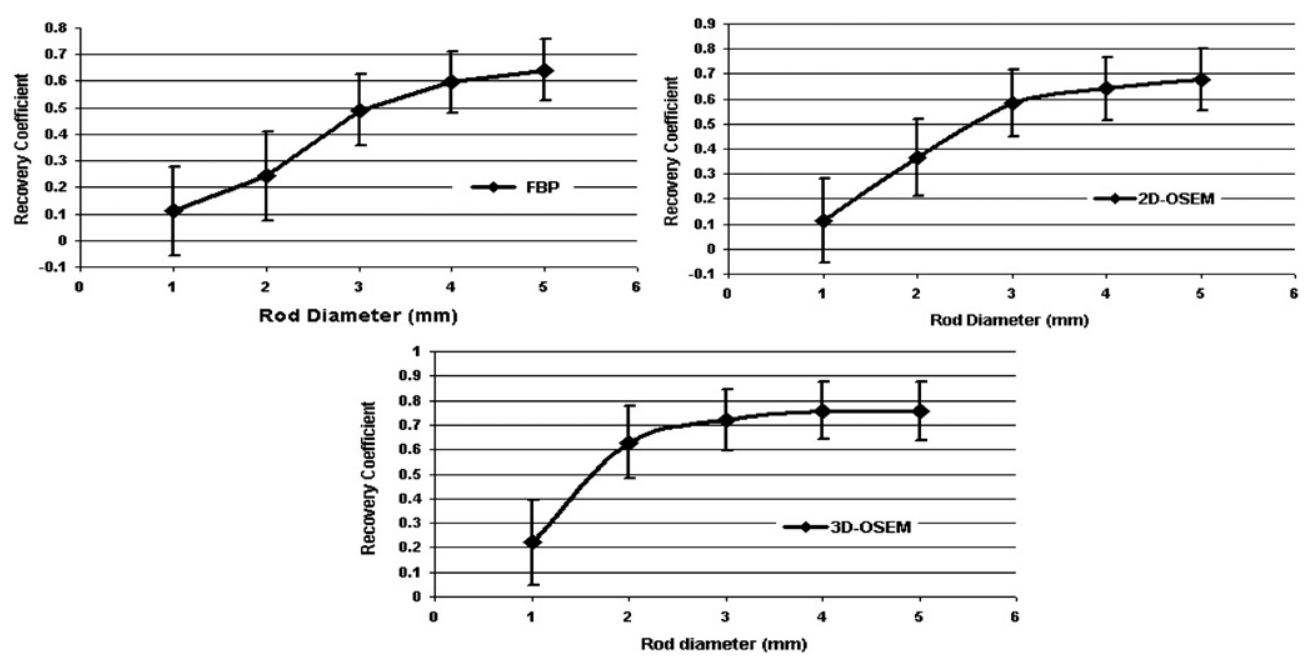

Figure 6. Recovery coefficients and standard deviation measured using the three reconstruction algorithms available with the $\mathrm{VrPET}$ scanner.

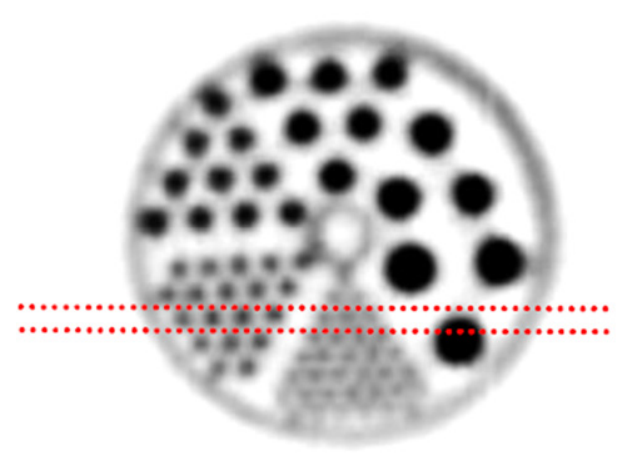

(a)

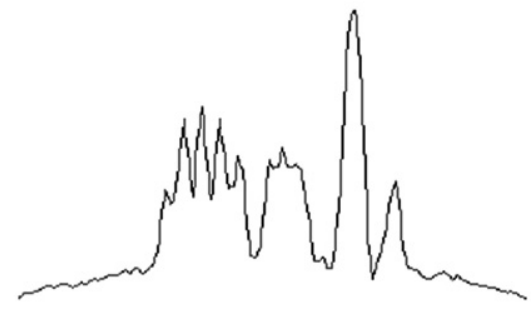

(c)

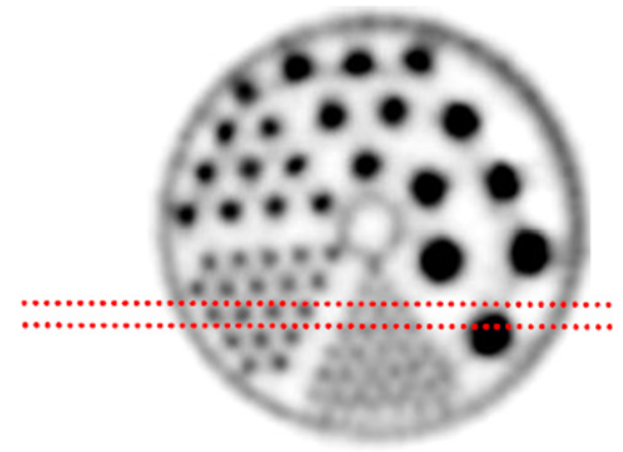

(b)

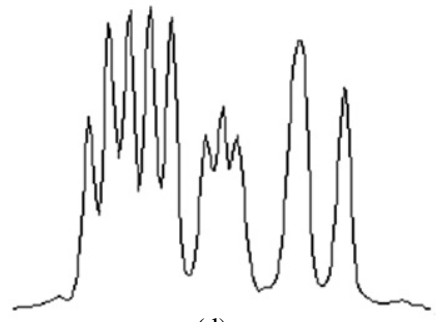

(d)

Figure 7. Transversal images of the Derenzo phantom reconstructed using two different reconstruction methods: SSRB+2D FBP (a) and 3D OSEM (b). The images were obtained by averaging 20 slices. The dotted lines indicate the position of the integrated profiles shown on bottom panels (c) and (d).

diameters in cold solid background are used in this phantom. Standard deviation of these recovery coefficients is also shown in figure 6 for each rod. The spillover ratios obtained for the water- and air-filled inserts of the IQ phantom as well as the standard deviation calculated in the same manner as that of the recovery coefficients (equation (2)) are reported in table 3. Since corrections for scatter are not applied, these values are indicative of the effect of scattered radiation on the reconstructed images. 

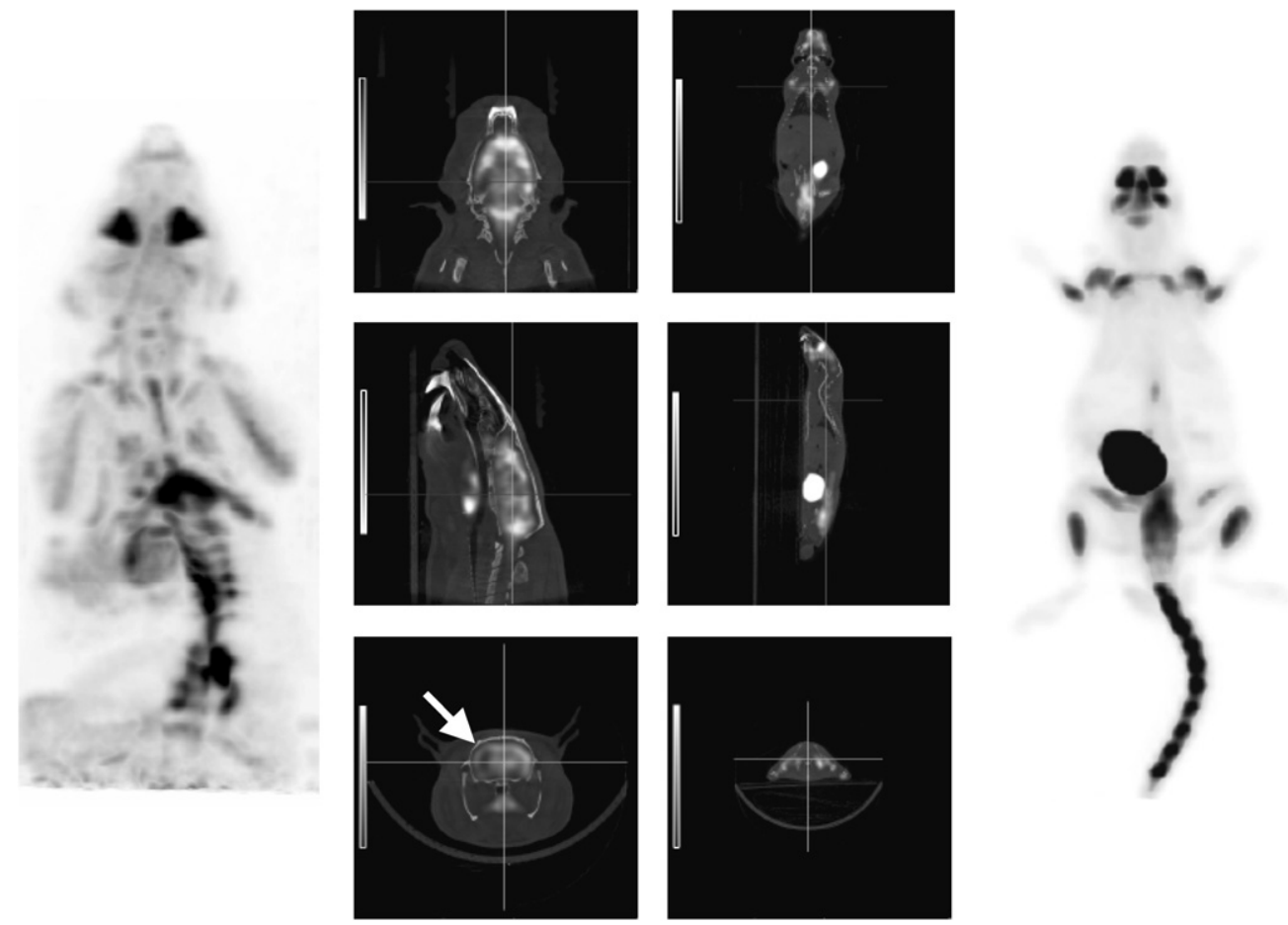

Figure 8. PET image MIP render of rat (left), mouse (right) and fused PET/CT slices (central columns). In the rat fused image (axial view), an area without FDG uptake in the left hemisphere of the brain is clearly visible, corresponding to the surgically induced ischemia.

Table 3. Spillover ratios and standard deviation (\%).

\begin{tabular}{lllll}
\hline & Water & $\%$ Std & Air & $\%$ Std \\
\hline FBP & 0.093 & 34.6 & 0.16 & 28.4 \\
2D OSEM & 0.085 & 27.5 & 0.13 & 19.17 \\
3D OSEM & 0.085 & 25.3 & 0.12 & 19.05 \\
\hline
\end{tabular}

\subsection{Phantom and animal studies}

Figure 7 shows transaxial views of the Derenzo phantom, corresponding to 20 averaged slices. These data were reconstructed using standard SSRB + FBP + ramp filtering (a) and 3D OSEM with 40 subsets and a single iteration (b). All hot spots are visible, and even the smallest rods $(1.2 \mathrm{~mm})$ are almost resolved in the image although the contrast is reduced relative to the larger rods due to partial volume effects. Figure 8 shows maximum intensity projection (MIP) views of a Wistar rat and a BALB/c mouse respectively. These images illustrate the ability of the VrPET/CT system to obtain high-resolution images of living rodents. Additionally, fused PET/CT slices of these studies are shown in central columns. The rat fused image is focused on the head, where an area without FDG uptake is clearly visible in the left hemisphere of the brain, corresponding to the surgically induced ischemia.

\subsection{Inter-modality alignment}

After measuring the inter-modality alignment accuracy using the ${ }^{22} \mathrm{Na}$ sources included in the phantom, we found that the average mean squared error was $0.212 \pm 0.075 \mathrm{~mm}$ for the five 
tested configurations. These results indicate that inter-modality alignment error is less than half the PET resolution and the method employed has high repeatability.

\section{Discussion and conclusion}

A rotatory PET/CT scanner has been developed and its performance has been thoroughly evaluated. The evaluation is structured according to the recently approved NEMA NU-4 2008 recommendations, which are meant to facilitate the comparison between small-animal PET scanners.

The spatial resolution obtained is comparable with that reported for other commercially available small-animal dedicated tomographs (Knoess et al 2003, Del Guerra et al 2006, Wang et al 2006, Huisman et al 2007, Yamada et al 2008, Bergeron et al 2007, Bao et al 2009). The absolute sensitivity was $2.22 \%$ for an energy window of $100-700 \mathrm{keV}$, which is a reasonable value for a partial-ring system. The count rate performance measurements indicate that the scanner is well suited for imaging mice and rats using low activity concentrations. However, figure 5 shows an onset of significant dead-time effects, which affect the maximum NEC rate obtainable. The relative system sensitivity to scattered radiation $(11.45 \%$ for mouse-sized objects and $23.26 \%$ for rat-sized objects) is within reasonable limits, but a direct comparison with other scanners is hampered by the difference in the method of deriving SF values. To the authors' best knowledge, there is only one performance evaluation carried out using this protocol (Bao et al 2009).

In order to convert measured image pixel values to activity concentrations with the best achievable accuracy, additional corrections for scatter and attenuation are needed, especially when imaging cold lesions due to the increased presence of scattered radiation shown by the spillover ratios. Some of these corrections could be derived from the CT images generated by the CT module of the scanner. With regard to the workflow design, although the suitability of similar configurations has been demonstrated for simultaneous metabolic and anatomic imaging of small animals (Goertzen et al 2002), the VrPET/CT is intended to acquire these modalities sequentially. This design specification was derived from the fact that the most commonly used CT data acquisition protocol, intended to provide anatomical templates for the PET images, is time inexpensive (up to $1.5 \mathrm{~min}$ per bed position) when compared with the PET scan times.

The inter-modality alignment accuracy is less than half the PET resolution and the method employed has demonstrated high repeatability. Additionally due to the coplanar layout of this system, alignment errors in the axial dimension due to CT magnification are minimized due to the mechanical alignment of both fields of view.

This work demonstrates the feasibility of a coplanar PET/CT system for in vivo imaging of small laboratory animals. The physical configuration of this system provides intrinsically coregistered datasets; thus, it is not necessary to reposition the animal to perform multimodality imaging. Although there is still some room for improvement, such as the above-mentioned dead time, the performance values obtained using this configuration makes this scanner suitable for PET applications, while the cost derived from detectors and electronics is significantly reduced in this design as compared with a full-ring configuration PET tomograph.

\section{Acknowledgments}

The authors thank SEDECAL Engineering Department for their support and the development of the mechanical parts and to Alexandra de Francisco for assistance with the animal 
studies. This project was supported by the CENIT Program (Ministerio de Industria), CIBER CB07/09/0031 (Ministerio de Sanidad y Consumo), TEC2007-64731/TCM and TEC200806715-C02-01 (Ministerio de Educación y Ciencia).

\section{References}

Abella M, Vaquero J J, Vicente E, Álvarez J, Lage E and Desco M 2006 Effect of misalignments in small animal positron emission tomography scanners based on rotating planar detectors Mol. Imaging Biol. 8 75-6

Bao Q, Newport D, Chen M, Stout D B and Chatziioannou A 2009 Performance evaluation of the inveon dedicated PET preclinical tomograph based on the NEMA NU-4 standards J. Nucl. Med. 50 139-47

Berard P, Pepin C M, Rouleau D, Cadorette J and Lecomte R 2005 CT acquisition using PET detectors and electronics IEEE Trans. Nucl. Sci. 52 634-7

Bergeron $\mathrm{M}$ et al 2007 Performance evaluation of the LabPET ${ }^{\mathrm{TM}}$ APD-based digital PET scanner IEEE NSS/MIC 2007 Conf. Record vol 6 pp 4185-91

Cañadas M, Embid M, Lage E, Desco M, Vaquero J J and Perez J M 2008 Performance comparison of two commercial Small Animal PET Scanners: ClearPETTM and rPET-1TM IEEE NSS/MIC 2008 Conf. Record pp 4773-9

Cherry S R, Louie A Y and Jacobs R E 2008 The integration of positron emission tomography with magnetic resonance imaging Proc. IEEE 96 416-38

Del Guerra A et al 2006 Performance evaluation of the Fully Engineered YAP-(S)PET Scanner for Small Animal Imaging IEEE Trans. Nucl. Sci. 53 1078-83

Fontaine R, Belanger F, Cadorette J, Leroux J D, Martin J P, Michaud J B, Pratte J F, Robert S and Lecomte R 2005 Architecture of a dual-modality, high-resolution, fully digital positron emission tomography/computed tomography (PET/CT) scanner for small animal imaging IEEE Trans. Nucl. Sci. 52 691-6

Goertzen A L, Meadors A K, Silverman R W and Cherry S R 2002 Simultaneous molecular and anatomical imaging of the mouse in vivo Phys. Med. Biol. 47 4315-28

Herráiz J L, España S, Vaquero J J, Desco M and Udías J M 2006 FIRST: fast iterative reconstruction software for (PET) tomography Phys. Med. Biol. 51 4547-65

Huisman M C, Reder S, Weber A W, Ziegler S I and Schwaiger M 2007 Performance evaluation of the Philips MOSAIC small animal PET scanner Eur. J. Nucl. Med. Mol. Imaging 34 532-40

Knoess C et al 2003 Performance evaluation of the microPET R4 PET scanner for rodents Eur. J. Nucl. Med. Mol. Imaging 30

Lage E, Vaquero J J, Redondo S, Abella M, Tapias G, Udías A and Desco M 2006 Design and development of a high performance micro-CT system for small-animal imaging IEEE NSS/MIC 2006 Conf. Record pp 3549-52

Liang H, Yang Y, Yang K, Wu Y, Boone J M and Cherry S R 2007 A micro PET/CT system for in vivo small animal imaging Phys. Med. Biol. 52 2881-3894

Meei-Ling J, Yu-Ching N, Kuo-Wei C, Hsing-Ching L, Keh-Shih C and Ying-Kai F 2006 A combined micro-PET/CT scanner for small animal imaging Nucl. Instrum. Methods Phys. Res. A 569 314-8

Nassalski A, Moszynski M, Syntfeld-Kazuch A, Swiderski L, Szczesniak T, Wolski D and Batsch T 2008 Application of Hamamatsu S8550 APD array to the common PET/CT detector IEEE Trans. Nucl. Sci. 55 2460-4

NEMA 2008 Performance measurements for small animal positron emission tomographs NEMA Standards Publication NU 4-2008, Technical Report (Rosslyn, VA: National Electrical Manufacturers Association)

Ortuño J E, Guerra P, Rubio J L, Kontaxakis G and Santos A 2006 3D-OSEM iterative image reconstruction for high-resolution PET using precalculated system matrix Nucl. Instrum. Methods Phys. Res. A 569 440-4

Ortuño J E, Rubio J L, Guerra P, Kontaxakis G, Desco M, Vaquero J J and Santos A 2008 Efficient methodology for 3D statistical reconstruction of high resolution coplanar PET/CT scanner IEEE NSS/MIC 2008 Conf. Record pp 5096-100

Pascau J, Vaquero J J, Abella M, Cacho R, Lage E and Desco M 2006 Multimodality workstation for small animal image visualization and analysis Mol. Imaging Biol. 8 97-8

Phelps M E 2000 Positron emission tomography provides molecular imaging of biological processes Proc. Natl Acad. Sci. USA $979226-33$

Pomper M G 2001 Molecular imaging: an overview Acad. Radiol. 8 1141-53

Raylman R R, Majewski S, Velan S S, Lemieux S, Kross B, Popov V, Smith M F and Weisenberger A G 2007 Simultaneous acquisition of magnetic resonance spectroscopy (MRS) and positron emission tomography (PET) with a prototype MR compatible, small animal PET imager J. Magn. Reson. 1 305-10

Riendeau J, Bérard P, Viscogliosi N, Tétrault M A, Lemieux F, Lecomte R and Fontaine R 2008 High rate photon counting CT using parallel digital PET electronics IEEE Trans. Nucl. Sci. 55 40-7 
Rodriguez-Ruano A, Pascau J, Chamorro J, Sisniega A, García-Vázquez V, Udias A, Vaquero J J and Desco M 2008 PET/CT alignment for small animal scanners based on capillary detection IEEE NSS/MIC 2008 Conf. Record pp 3832-5

Siegel S, Aloj L, Seidel J, Jagoda E, Gandler W, Eckelman W and Green M 1999 Initial results from a PET/planar small animal imaging system IEEE Trans. Nucl. Sci. 46 571-6

Townsend D W and Beyer T 2002 A combined PET/CT scanner: the path to true image fusion Br. J. Radiol. S24-S30

Vaquero J J, Lage E, Ricon L, Abella M, Vicente E and Desco M 2005 rPET detectors design and data processing IEEE NSS/MIC 2005 Conf. Record 5 2885-9

Vaquero J J, Redondo S, Lage E, Abella M, Sisniega A, Tapias G, Soto-Montenegro M L and Desco M 2008 Assessment of a new high-performance small-animal x-ray tomograph IEEE Trans. Nucl. Sci. 55 898-905

Vaquero J J, Seidel J, Siegel S, Gandler W R and Green M V 1998 Performance characteristics of a compact position-sensitive LSO detector module IEEE Trans. Med. Imaging 17 967-78

Wang Y, Seidel J, BMW T, Vaquero J J and Pomper M G 2006 Performance evaluation of the GE healthcare eXplore VISTA dual-ring small-animal PET scanner J. Nucl. Med. 47 1891-900

Weissleder R 2002 Scaling down imaging: molecular mapping of cancer in mice Nat. Rev. Cancer 2 11-8

Yamada R et al 2008 Development of a small animal PET scanner using DOI detectors IEEE Trans. Nucl. Sci. 55 906-11

Zingone A, Seidel J, Aloj L, Caraco C, Vaquero J J, Jagoda E M, Chou J Y, Green M V and Eckelman W C 2002 Monitoring the correction of glycogen storage disease type 1a in a mouse model using [(18)F]FDG and a dedicated animal scanner Life Sci. 71 1293-301 